\title{
The Role of Aspiration and Thyroxine Suppression Therapy in Management of Benign Thyroid Nodules
}

\author{
Jalil Ibrahim Awaid alrubaye'; Hussein Ali Abed Ahmad ${ }^{2}$; Salah Mahdi Tager ${ }^{3}$ \\ ${ }^{1}$ MBCHB, FICMS) Department of Surgery/Al -Zahra Hosp Al-Kut; ${ }^{2}$ Assist prof. Wassit Medical Collage M. B. Ch. \\ B- F.I.C.M.S ; ${ }^{3}$ Consulltant General Surgeon(MBCHB.CABS)
}

\begin{abstract}
A prospective study on 142 patients presenting with (benign euthyroid nodule), after diagnostic work-up included a clinical evaluation, thyroid function tests, ultrasonography, thyroid scintigraphy and fine needle aspiration cytology. Based on the investigations, patients were managed with aspiration andthyroxine suppression therapy for sex month. Patients were divided into four groups according to their response of treatment, group( 1) complete shrinkage response, group( 2) reduction in size, group (3) no response but same size and group (4) enlarge in size non responders'. Of the 142 patients17 (12\%) were male, and 125 $(88 \%)$ were female. The age rangein $99(69.7 \%)$ patients were between 21 and 50 years and forty three patients were above 50 years of age. We divided our result into four groups,group( 1) complete shrinkage response29 patients' (20.5\%),group( 2) reduction in size 45 patients' (31.8\%),group (3) no response but same size38 patients' $(26.7 \%)$ and group (4) enlarge in size non responders30 patients'(21\% )Thyroxine suppressive treatment were effective in shrinking about one fifth of thyroid nodules. Similarly, there was reduction in size in about one third of nodule.From 17 male 6 patients $(35 \%)$ achieve complete response ,while only 23 female patients (18\%) achieve complete response, and from 17 male patients 5 (29\%) achievereduction in size, while only 40 female patients (32\%) achieve reduction in size. Young age less than fifty achieve good response than older patients(63.6\%). Single aspiration needed in 26 patients( $18.3 \%)$ while multiple aspiration needed in 116 patients(81.7\%).
\end{abstract}

Key words : Aspiration, Thyroid Nodules, thyroxine suppression treatment.

\section{Introduction}

Medical management of benign thyroid nodules may deter the requirement for medical procedure and morbidity related to it. Thyroxine inhibition treatment is being used in the medicinal management of euthyroid benign nodules ${ }^{12}{ }^{2}$. Nodular thyroid infection still one of the common endocrine troubles. The majority thyroid nodules are benevolent hyperplastic injury however 5 to $20 \%$ of thyroid nodules are genuine neoplasm ${ }^{3}$. The predominance of thyroid nodules differs impressively relying upon an assortment of elements that incorporate iodine consumption inside a given populace, age, sex, diet, drug and natural radiation presentation.

The age-related is increment the nodularity and thyroid volume. Little thyroid nodule are ordinarily found in patients with a simultaneous history of Hashimoto's Thyroiditis ${ }^{4}$. The significance of dealing with a thyroid nodule in the early separation among benign and harmful nudule, since the way to deal with treatment in the two is thoroughly different. The viability of thyroxine concealment treatment in benign euthyroid nodule brings about the justification of medical procedure in such patients.

Most investigations have demonstrated that a little of thyroid nodule relapse in patients treatment with thyroid hormone. Nonetheless, the suppressive treatment appears to intervene with goitrogenesis in numerous patients, and recent hypothesis proposes that it could diminish the danger of thyroid oncogenesis ${ }^{5}$.

Inhibition of thyroid-stimulation hormone(TSH) discharge in ordinary subjects by the giving of thyroid hormone brings about thyroid decay ${ }^{6}$,even though the pathogenesis of thyroid nodule and irregular nontoxic multinodular goiters is ineffectively comprehended, TSH is dared to be essential if not adequate and, along these lines, inhibition of TSH secretion may be relied upon to bring about a diminishing in nodule or goiter size or possibly avert further augmentation. 
The significance of TSH in goiter production changes with the reason for the goiter. For instance, in patients with iodine inadequacy or autoimmune disease (Hashimoto's), an elevated in TSH secretion is the dominating reason for goiter. Conversely, the majority patients with thyroid nodule or irregular nontoxic multinodular goiters have typical serum TSH levels, especially persons with nontoxic multinodular goiters, the thyroid expansion is most likely caused by many factors (include TSH) that work after some time on thyroid follicular cells that have special manufactured and growth possibilities. The effect is spread and presently multinodular thyroid augmentation; a few nodule inevitably become independent ${ }^{7}$ and others may experience cystic deterioration. Since the thyroid hormone is assumed to decrease goiter size by diminishing TSH emission, inhibition treatment would be relied upon to be ineffectual in patients in whom plasma TSH levels were previously subnormal because of autonomous thyroid hormone creation ${ }^{8}$

\section{Patients and Methods}

The research was carried out in AL-Zahra teaching hospital from May 2011 until May 2015, on 142 patients presenting with (benign euthyroid nodule), after diagnostic work-up included a medicalevaluation by history information, write of present symptoms with their period, a systemic assessment and local assessment of the thyroid enlargement and the neck. The quantitative determination of plasma triiodothyronine(T3) and plasma thyroxine(T4) was carried out by radioimmunoassay(RIA), (normal values of T3: 0.72-2.2 $\eta \mathrm{g} / \mathrm{ml}, \mathrm{T} 4:$ 5.6-13.6 $\mu \mathrm{g} / \mathrm{ml})$. Serum TSH was measured by the immunoradiometric assay (IRMA), (normal range 0.3-6.5 $\mu \mathrm{IU} / \mathrm{ml}$ ). Fine Needle Aspiration Cytology( FNAC) was performed as therapeutic and diagnostic, result were write as thyroid cyst. According to the earlymedical, biochemical assessment, FNAC and thyroid examine. The subsequentsubjects were excluded from this study: Goiter hypothyroidism, hyperthyroidism, pre-pubertal and pubertal, distribute nodular goiter and suspicious FNAC result.

All included patients after aspiration given thyroxine suppression at a dose of $50-100 \mu \mathrm{g}$ every day. TSH was reserved in the littletypical range for 6 months. subjects were followed up every month to observe the size of the nodules by medical palpation and ultra-sonography also repeated aspiration was performed for those with persistent nodule. Patients were divided into four groups according to their response of treatment group(1) complete shrinkage response, group(2) reduction in size ,group (3) no response but same size and group (4) enlarge in size non responders'. Operation was performed in states where the nodule did not retreatfollowing6 months of thyroxininhibition.

\section{Findings}

Of the 142 patients17 (12\%) were male, and $125(88 \%)$ were female. The median age was 37 years Ninety nine $(69.7 \%)$ patients were between 21 and 50 years and forty three $(30.3 \%)$ patients were above 50 years of age. We divided our result into four groups, group( 1) complete shrinkage response 29 patients'(20.5\%),group( 2) reduction in size 45 patients' (31.8\%),group (3) no response but same size38 patients' (26.7\%) and group (4) enlarge in size nonresponders30 patients' $(21 \%)$ Thyroxin suppressive treatment were effective in shrinking about one fifth of thyroid nodules. Similarly, there was reduction in size in about one third of nodule. From 17 male 6 patients (35\%) achieve complete response, while only23 female patients (18\%) achieve complete response, and from 17 male patients $5(29 \%)$ achieve reduction in size, while only 40 female patients (32\%) achieve reduction in size. From 99 Young age patients(less than fifty years) 63 patients(63.6\%) achieve good response either complete response 25 patients $(25.2 \%)$ or reduction in size 38 patients( $38.3 \%$ ), while from 43 older patients 11 patients $(25.6 \%)$ achieve good response either complete response 4 patients $(9.3 \%)$ or reduction in size 7 patients(16.2\%). The common side effect areweight loss 7 patients(4.9\%) , insomnia4 patients(2.8\%) and palpitation 16 patients $(11.2 \%)$ but all patients tolerate these side effectby reduction the dose.Single aspiration needed in6 patients(4.2\%) while multiple aspiration needed in 136 patients( $95.8 \%$. Group 3 and 4( 68 patients $47.8 \%$ ), there was no response in spite of aspiration and thyroxin suppressionunderwent subtotal thyroidectomy. 
Table (1) Response groups and Sex.

\begin{tabular}{|l|l|l|}
\hline Response & Male & Female \\
\hline ( 1) Complete Shrinkage & 6 & 23 \\
\hline ( 2) Reduction in Size & 5 & 40 \\
\hline (3) Same Size & 3 & 35 \\
\hline (4) Enlarge in Size & 3 & 27 \\
\hline
\end{tabular}

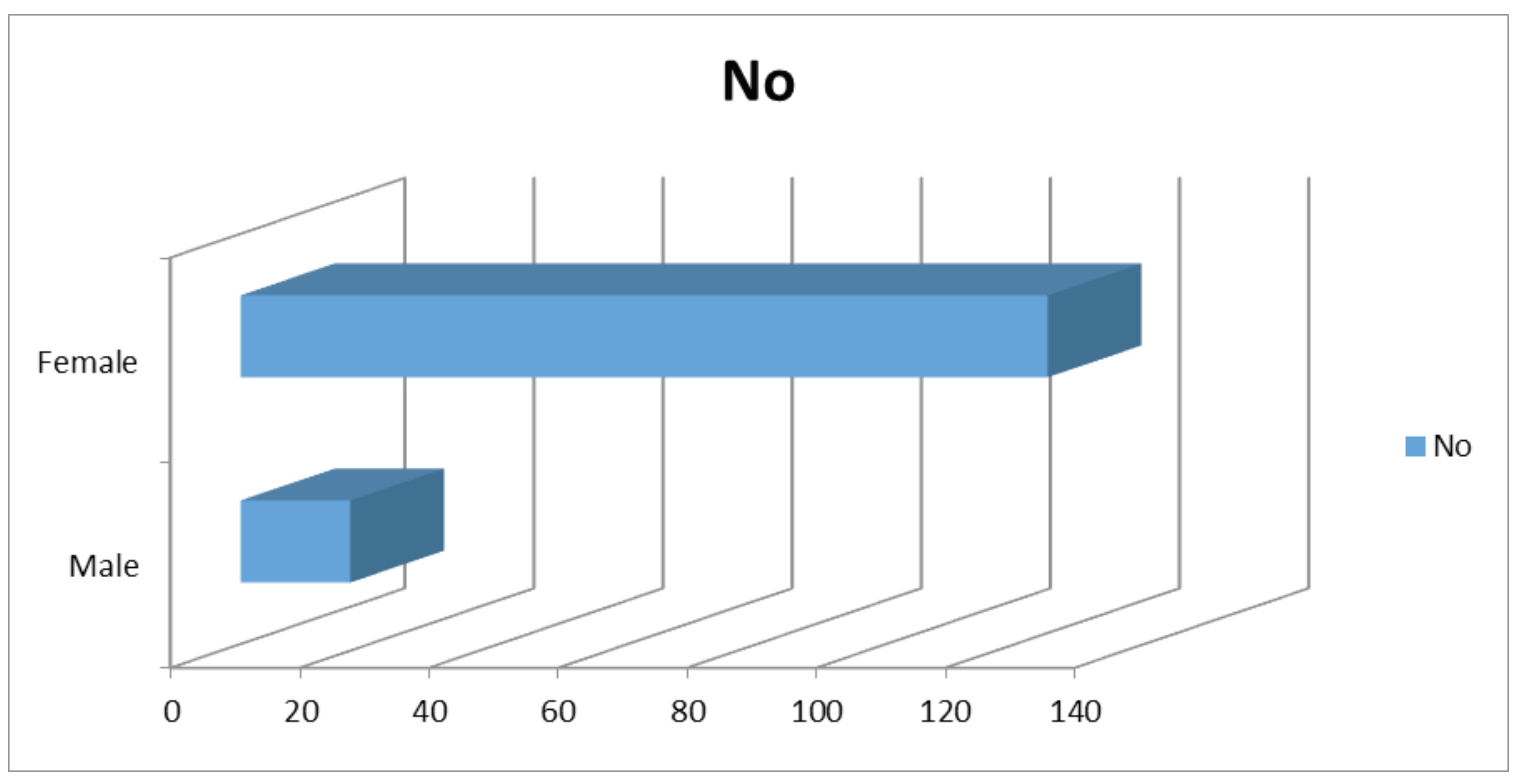

Figure (1) number of patients sex.

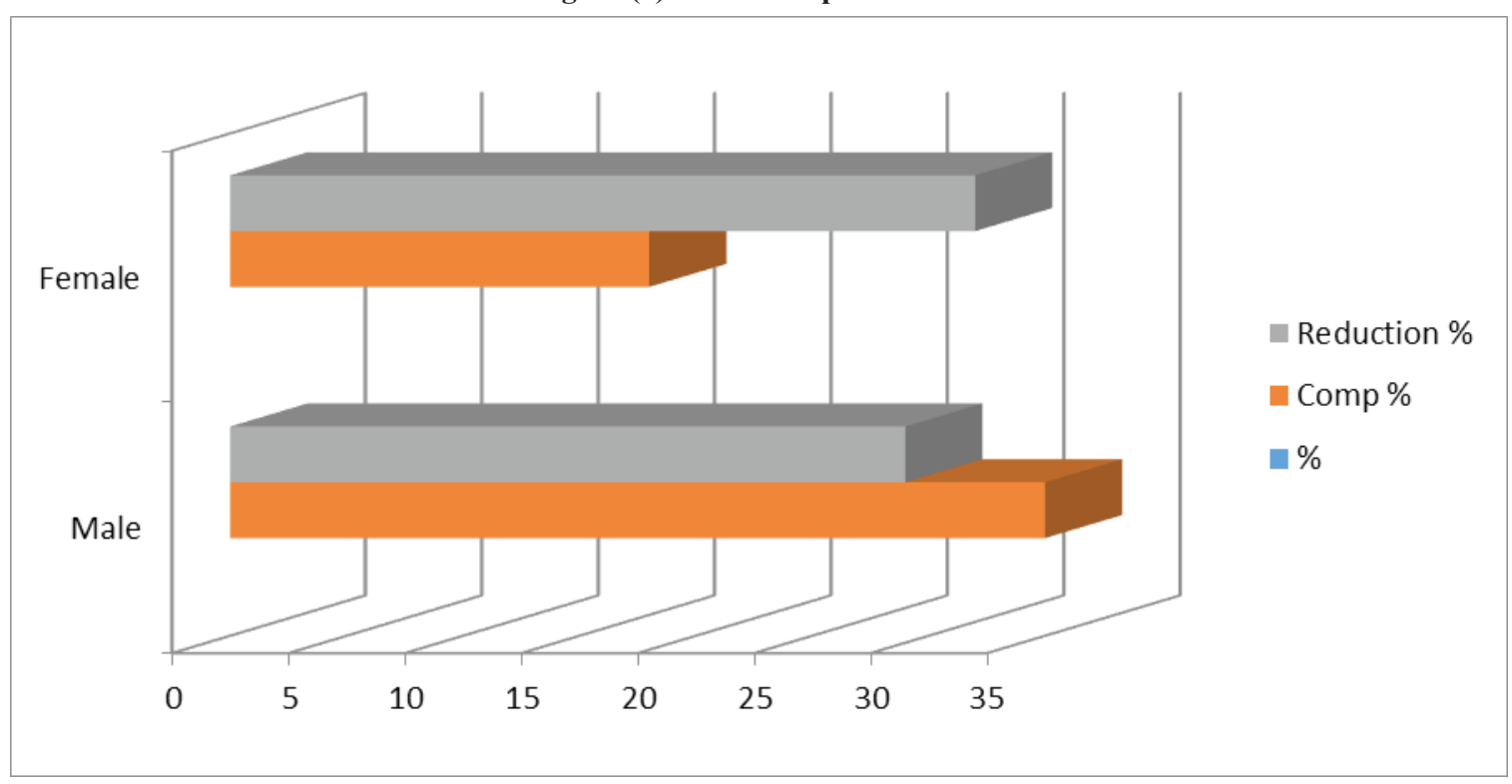

Figure (2) patient sex and response to thyroxine suppression therapy. 


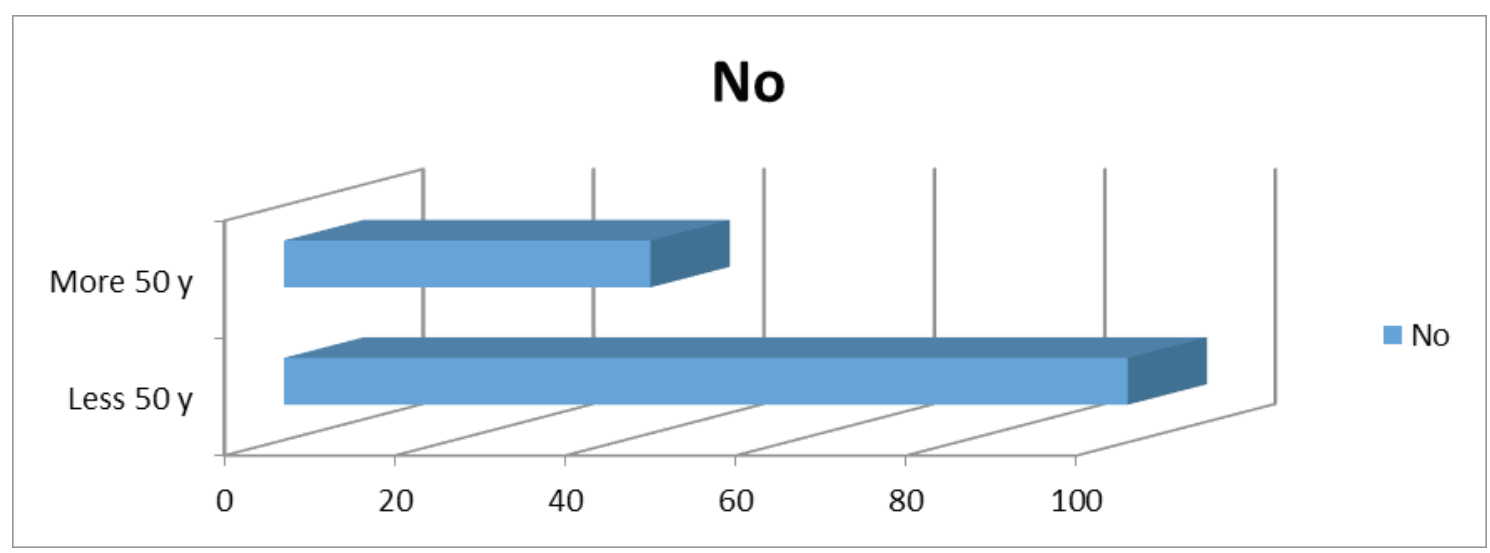

Figure (3) patients age distribution .

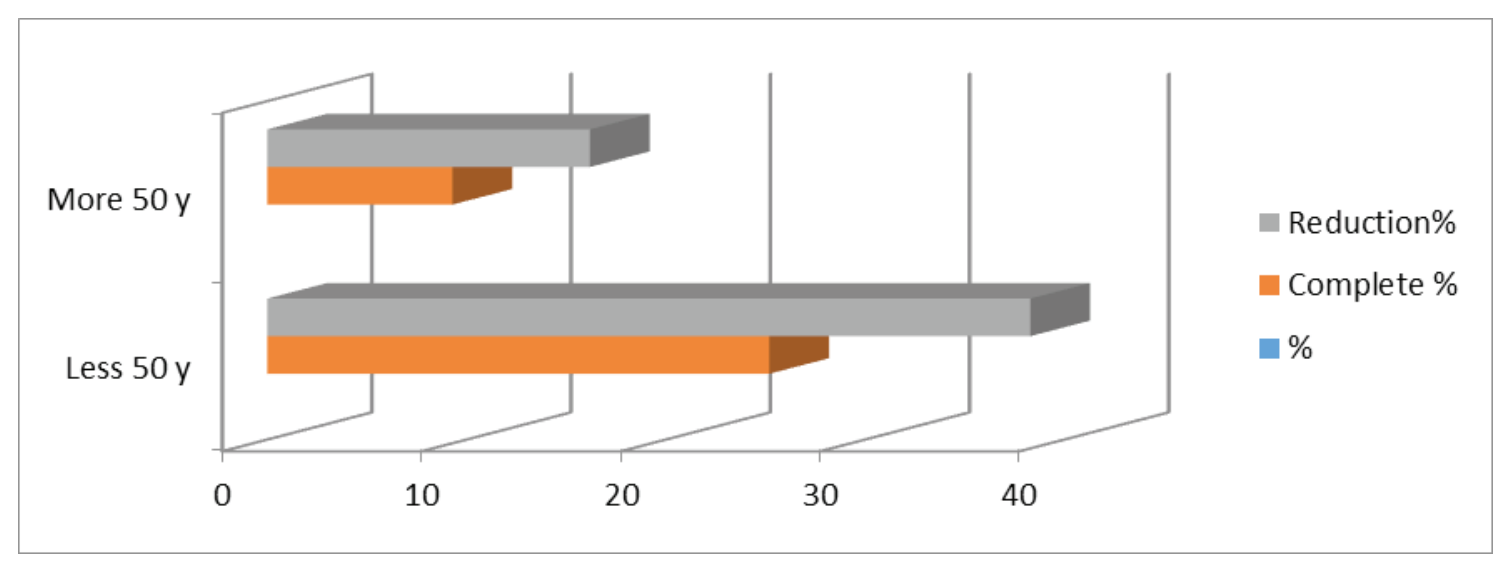

Figure (4) patients age and response to thyroxine suppression therapy.

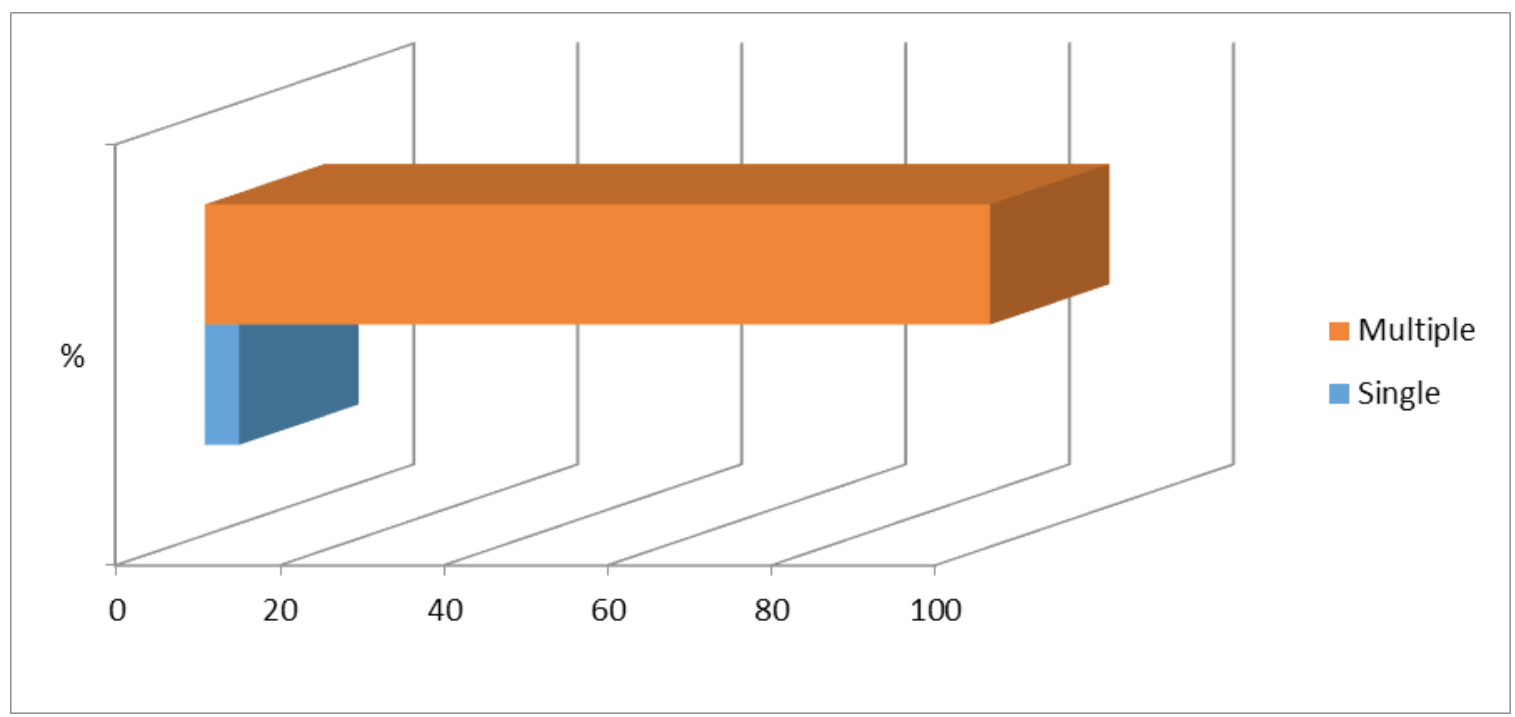

figure (5) the percentage of aspirationneeded 


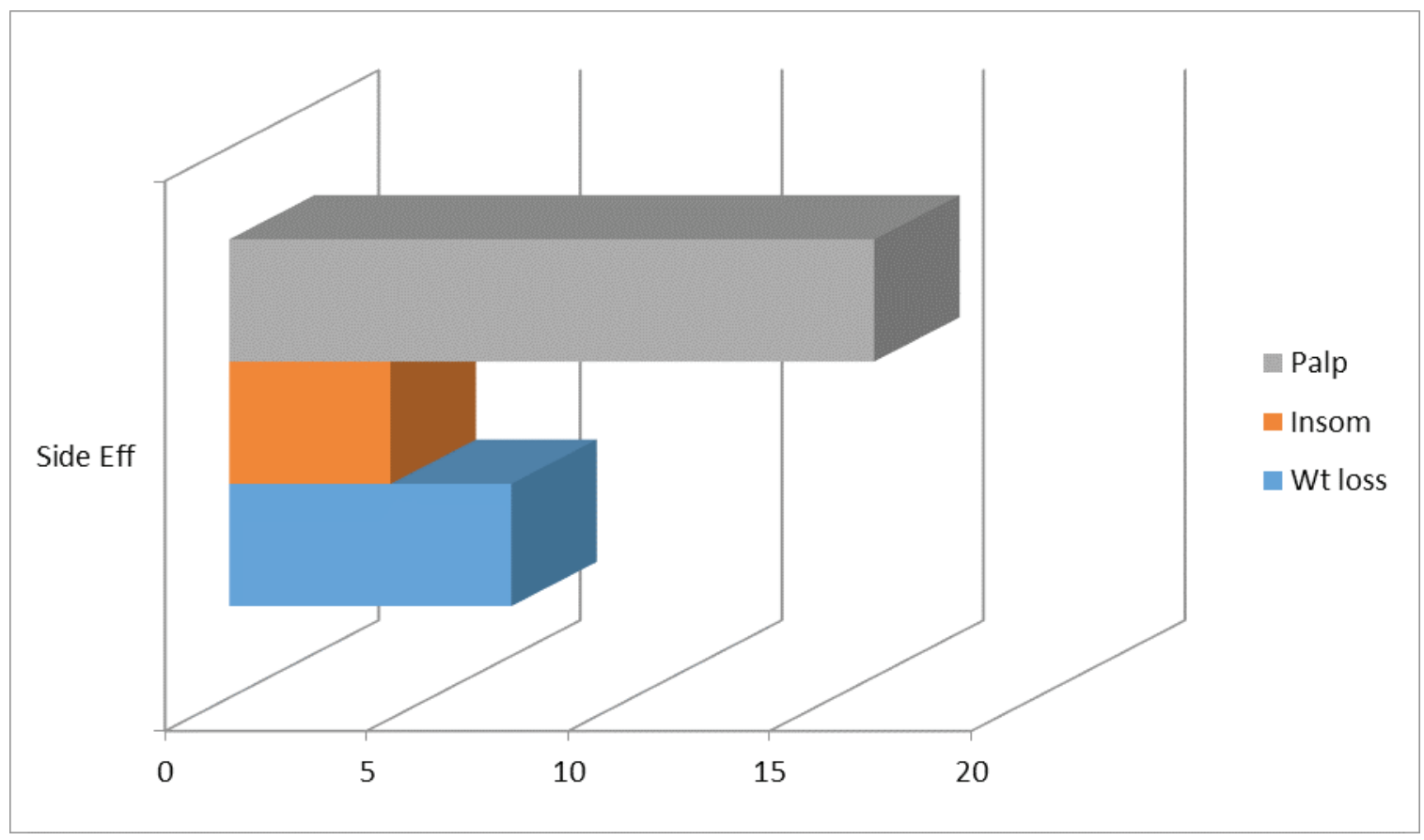

Figure (6) The common side effect

\section{Discussion}

The American Association of Clinical Endocrinologists(AACE) does not containthyroxininhibition in its advice for running of benign euthyroid nodule ${ }^{8}$ and restricts its use to patients with FNAC negative thyroid nodules not from iodinepoorregion, youthfulpersons with little goiter and nodules with no confirmation of practical autonomy. It additionalconditions that thyroxine inhibitionmust be avoided in big goiter, clinically doubtfulinjury or those with insufficient cytologic example, in postmenopausal females or persons with osteoporosis and cardiovascular disease. However, thyroid hormone treatment for nodular goiter, for superficial reasons or to avoid investigative and healingoperation surgery, results in reasonable to totalweakening of the nodule ${ }^{9}$.Zygmunt, reports that in the early stage hyperplasic goiter may regress if thyroxine is given at a dose of $0.15-0.2 \mathrm{mg}$ daily for a few months ${ }^{10}$.

Thyroxine inhibitiontreatment with aspiration is an suitablesubstitute as long as the person is followedup cautiously at 3-month period because fluid may be re accumulated ${ }^{11}$.It is set in dosageenough to inhibit the TSH value to $0.1-0.5 \mu \mathrm{U} / \mathrm{ml}$ for 6 months; additionalextendedtreatment is kept for persons in whom a reduce in nodule size is recognized on USG. following 12 months, the dosage of thyroxine is reduced to preserve the plasmalevel of TSH in the little normal value. information also propose that thyroxine inhibition for benign nodules does not decisively result in decline of nodules, while a littleexamination of inhibition may be sensible in special patients ${ }^{12}$.A study by La Rosa ${ }^{13}$ has shown a $35 \%$ reduction in the size of the colloid and regenerative nodules. In our study, we achieve shrinkage in about one-fifth of thyroid nodules $(20.5 \%)$, Similarly, there was a reduction in size in about onethird of nodule $(31.8 \%)$ at a response rate of $52,3 \%$. In our study 17 patients (12\%) were male, and $125(88 \%)$ were female, Figure (1). These because that every kinds of simple goiter are extrafrequent in women than menas a result of the found of estrogen receptors in thyroid tissues $^{10}$.

The age range in 99 patients $(69.7 \%)$ were between 21 and 50 years and 43 patients $(30.3 \%)$ were more than 50 years of age Figure (3). In a review article, Mazzaferri ${ }^{14}$ reports an elevated in the occurrence of thyroid nodules with age and a occurrence of 5\% in persons at 50 years present by physical palpation. The ultrasonography (USG noticeoccurrence is far elevated, approximately $55 \%)^{15}$. Among the response groups, From 17 male 6 patients (35\%) achieve complete response, while only23 female patients $(18 \%)$ achieve complete response, 
and from 17 male patients 5 (29\%) achieve reduction in size, while only 40 female patients (32\%) achieve reduction in size Figure (2). From 99 Young age patients (less than fifty years) 63 patients(63.6\%) achieve good response either complete response 25 patients(25.2\%) or reduction in size 38 patients(38.3\%), while from 43 older patients 11 patients(25.6\%) achieve good response either complete response 4 patients( $(9.3 \%)$ or reduction in size 7 patients(16.2\%) Figure (4). We conclude that the younger age group of our patients due to the iodine deficiency which endemic in our country and most of our patients were fertile women.

We achieve good responses in the male group because of the absence of the estrogen receptor in thyroid tissue that not competent with thyroxine therapy ${ }^{10}$. Aspiration and FNAC have good sensitivity and a specificity in the exclusion of suspicious nodules ${ }^{16}$, but single aspiration was needed in 6 patients(4.2\%) while multiple aspirations needed in 136 patients(95.8\%) Figure(5) these mean that aspiration was not significant than thyroxine and fluid may re-accumulate without thyroxine and benefit only in FNAC stay the basis of helping to identify suspicious nodule. In our study, no response but the same size in 38 patients' $(26.7 \%)$ and enlarge in size in 30 persons $(21 \%)$, despiterecurring aspiration and thyroxine inhibition. The response speed of $52.3 \%$ to aspiration and thyroxine inhibition in the current study was more than that in other studies, wherever it is write to level from $17 \%$ [15] to $35 \%$. [13]. The cause could be a larger number of younger persons $(63.6 \%)$ presented less than 50 years of age). The common side effect is weight loss 7 patients( $4.9 \%)$, insomnia 4 patients $(2.8 \%)$ and palpitation 16 patients (11.2\%) Figure (6) but all patients tolerate these side effects by reducing the dose.

\section{Conclusion}

Aspiration and thyroxine suppressive treatment were effective in shrinking thyroid nodule. Male patients achieve good response than female patients. The response is better in younger patients.

Conflict of Interest : none

Source of Funding : self

Ethical Clearance : From patients and my college.

\section{References}

1. Celani MF. Levothyroxine suppressive therapy in the medical management of non-toxic benign multinodular goiter. Clin Endocrinol. 1993;101:326-32.

2. Gharib H, James EM, Charboneau JW, Naessens JM, Offord KP, Gorman CA. Suppressive therapy with levothyroxine for solitary thyroid nodule: A double blind controlled study. N Eng J Med. 1987;17:70.

3. McCarty TM, Vassilopoulou-Sellin R, Lusting R, Lemont JP. Thyroid and Parathyroid Cancers. In: Pazdur R, Coia LR, Hoskins WJ, Wagman LD, editors. Cancer Management: A Multidisciplenary Approach. Melville: PR; 2004.

4. Greenspan FS. The problem of the nodular goiter. Med Clin North Am. 1991;75:195-209.

5. Papini E, Bacci V, Panunzi C, Pacella CM, Fabbrini $\mathrm{R}$, Bizzarri $\mathrm{G}$, et al. A prospective randomized trial of levothyroxine suppressive therapy for solitary thyroid nodules. Clin Endocrinol. 1993;38:507-13.

6. Rienhoff, WF Jr. Microscopic changes induced in thyroid gland by oral administration of desiccated thyroid. Arch Surg 1940; 41:487]..

7. Studer H, Peter HJ, Gerber H. Natural heterogeneity of thyroid cells: the basis for understanding thyroid function and nodular goiter growth. Endocr Rev 1989; 10:125.]

8. Cooper DS, Doherty GM, et al. Revised American Thyroid Association management guidelines for patients with thyroid nodules. 2009; 19:1167

9. American Association of Clinical Endocrinologists and Associazione Medici Endocrinologi Medical Guidelines for clinical practice for the diagnosis and management of Thyroid Nodules-AACE/AME Task Force on thyroid Nodule. Thyroid Nodule Guideline. Endocr Pract. 2006;12:122.

10. Zygmunt H. Krukowshi, Norman S. Williams Christopher J.K.B., and P.Ronan OConnell. The Thyroid and Parathyroid Gland . Bailey \& Loves. Short Practice of Surgery 25th ed., Arnold, London, 2008, P.781

11. Hermus AR, Huysmans DA. Treatment of benign nodular thyroid disease. N Engl J Med. 1998;338:1438-47.

12. De Groot Leslie J, Larsen P.Reed. Churchill Livingstone: New York; Hennemann Georg 
Thyroid and its disease.

13. La Rosa GL, Ippolito AM, Lupo L, Cercabene G, Santonocito MG, Vigneri $R$, et al. Cold thyroid nodule reduction with L-thyroxine can be predicted by initial nodule volume and cytological characteristics. J Clin Endocrinol Metab. 1996;81:4385-7.
14. . Mazzaferi EL. Management of a solitary thyroid nodule. N Engl J Med. 1993;328:553-9.

15. Welker MJ, Orlov D. Thyroid nodules. Am Fam Physician. 2003;67:559-66.

16. Gharib H. Fine needle aspiration biopsy of thyroid nodules: Advantages, limitations and effect. Mayo Clin Prac. 1994;69:44-9. 